\title{
Cortical epileptogenesis of slowly kindled freely moving rats
}

\author{
Orbán-Kis Kª Száva Iringóa ${ }^{*}$, Szilágyi T \\ Department of Physiology, University of Medicine and Pharmacy Tirgu Mures, Romania
}

\begin{abstract}
Objective. Epilepsy is a neurological disorder that can be caused by many underlying pathologies. The epileptic and interictal manifestations that appear during the progression of chronic epilepsy are still not understood completely. One of the most frequent forms of this disease is temporal lobe epilepsy in which is clear involvement of the hippocampal formation. In order to study the electrografic progression of untreated seizures we used amygdala kindling in freely moving rats. Methods. Seven animals were implanted with bilateral hippocampal and prefrontal electrodes. A bipolar electrode, implanted in the lateral nuclei of the left amygdala was used for stimulation. The kindled group of animals was stimulated daily with the minimum current intensity needed to reach the afterdischarge threshold. Behavioral changes during kindling were scored according to the Racine scale. Results. The average seizure severity on the Racine scale was $2.6 \pm 0.4$ by day 6 and $4.4 \pm 0.6$ by day 20. The first spontaneous seizures appeared after 31 days of stimulation. During spontaneous seizures the preictal spike full width at half maximum increased gradually from $51 \pm 4 \mathrm{msec}$ to $110 \pm 5 \mathrm{msec}(p<0.05)$ whereas the amplitude of the negative field potential deflection increased by $62 \%(p<0.05)$. Conclusions. Our study showed that the progression of temporal lobe epilepsy, as seen in humans, can be reproduced in the kindling model with high fidelity. This study confirms in vivo the increase in preictal spike duration as well as the increase of the amplitude of negative field potential deflection during the preictal period.
\end{abstract}

Keywords: temporal lobe epilepsy, hippocampus, epileptogenesis, kindling

Received: 11 November 2014 / Accepted: 1 December 2014

\section{Introduction}

Epilepsy is a symptom of cerebral irritability [1] that can be caused among others by chanellopathies, cerebral malformations, brain traumatisms, brain tumors and brain infections [2]. Currently we do not understand completely the molecular changes, the morphological alterations or the clinical signs of epilepsy. Careful analysis of epileptic and interictal manifestations of epileptogenesis could provide useful data needed for understanding (1) the underlying mechanisms of chronic epilepsy progress, (2) the factors that influence its severity, and (3) how to prevent epileptogenesis if the initial insult already has occurred [3].

The hippocampal formation is of particular interest as it is involved in the encoding, consolidation, and retrieval of episodic memories [4] as well as in physiological and pathological oscillations [5]. One of the most frequent forms of epilepsy is temporal lobe epilepsy (TLE), in which the involvement of hippocampus seems extremely important [6].

The kindling phenomenon has been used as a chronic model of temporal lobe epilepsy for several decades [7] and it is accepted as a model of complex partial epilepsy with secondarily generalized seizures [8]. Kindling refers to a seizure-induced plasticity phenomenon that occurs when repeated afterdischarge induction by electrical stimulation in a specific brain region evokes a progressive development

\footnotetext{
a The first two authors had equal contribution

* Correspondence to: Károly Orbán-Kis

E-mail: orbank@gmail.com
}

of seizure susceptibility [7]; the seizures evoked by kindling closely resemble secondarily generalized partial seizures and are clearly progressive in nature [9]. Kindling helps to understand the consequences of untreated seizures, including mossy fiber sprouting, synaptic reorganization and progressive hippocampal neuronal loss [9].

The aim of the study was to monitor cortical electrogenesis in slowly kindled freely moving rats.

\section{Methods}

\section{Animals}

For the experiments we used male Wistar rats ( $\mathrm{n}=7$, weighing 250-380 g) that were housed individually, under standard conditions: constant temperature $\left(22-24^{\circ} \mathrm{C}\right)$ and humidity (50-60\%), 12 hours light/dark cycle and access to food and water ad libitum. All procedures involving animals were carried out in accordance with EU Directive 2010/63/EU and national and local guidelines and policies (CEC 26/2011).

\section{Electrode implantation and recording protocols}

Electrodes were implanted during surgery under general anesthesia (Ketamine $100 \mathrm{mg} / \mathrm{kg}$ and Xylazine $5 \mathrm{mg} / \mathrm{kg}$, i.m.). Body temperature was maintained at $38^{\circ} \mathrm{C}$ (Physiological Temperature Controller TMP-5b, Supertech, Pécs, Hungary) and vital signs were monitored continuously during the procedure. For stimulation purposes a stainless steel bipolar electrode (wire diameter 0.2, maximum distance between tips $0.3 \mathrm{~mm}$ ) was implanted in 
the lateral nuclei of the left amygdala $(5.5 \mathrm{~mm}$ lateral and $3.4 \mathrm{~mm}$ posterior from the bregma, depth $8 \mathrm{~mm}$ from the cerebral surface). For recording the propagation of ictal activity needle electrodes were implanted bilaterally in the CA3 region of the hippocampus (wire diameter $0.2 \mathrm{~mm}$, $2 \mathrm{~mm}$ lateral and $3 \mathrm{~mm}$ posterior from the bregma, depth $3.3 \mathrm{~mm}$ ) and $0-80 \times 1 / 16$ screw electrodes were fixed in the skull bilaterally above the frontal cortex $(3 \mathrm{~mm}$ anterior and $2 \mathrm{~mm}$ lateral from the bregma). 0-80x1/16 screw electrodes were fixed in the skull symmetrically above the cerebellum and used as ground and reference. Electrodes were connected to plastic connectors that were fixed on the skull with dental acrylic polymeric resin (electrodes and connectors were purchased from Plastics One Inc., Roanoke, VA, USA, dental acrylic resin was purchased from SpofaDental, Markova, Czech Republic). All electrodes were placed according to the stereotaxic atlas of Paxinos and Watson [10], the positioning was verified at the end of the protocol by histological procedures (Nissl staining).

The animals were monitored closely for 24 hours following the surgical procedure. After a postsurgical recovery period of 10-14 days a baseline electrical activity was recorded for 15 minutes with an 8-chanel amplifier (Multiamp SMA-4a, Supertech, Pécs, Hungary). All recordings were made on freely moving rats: animals were housed in a $50 \times 50 \mathrm{~cm}$ plexiglass cage, both connector plugs were connected through a $50 \mathrm{~cm}$ shielded cable to an overhead swivel $(6+2$ channels, Plastics One Inc, Roanoke, VA, USA) and then to the amplifier as well as the biological stimulator.

\section{Stimulation protocol}

The animals were divided in two groups: kindled $(n=4)$, and aged-matched sham-operated controls $(n=3$, to show that electrode implants are not epileptogenic in nature). Before starting the stimulation protocol we determined the epileptic threshold (afterdischarge threshold - ADT): first we used a train of 100 stimuli with $50 \mu \mathrm{A}$ amplitude, 0.1 -msec duration and $60 \mathrm{~Hz}$ frequency, then we gradually increased the current intensity in steps of $25 \mu \mathrm{A}$ up to $100 \mu \mathrm{A}$ and then by steps of $50 \mu \mathrm{A}$, until reaching the ADT. The kindled group of animals was stimulated daily through the implanted amygdala electrode with the minimum necessary current intensity to reach ADT and elicit seizures. In the control group only electroencephalography was recorded without stimulation. Daily stimulations were performed in each animal until the appearance of the first spontaneous seizures.

\section{Monitoring electrical and clinical seizures, data analy- sis}

Data recordings were performed using a 16 channel board (PCI 6036E National Instruments), at $1 \mathrm{kHz}$ resolution. In order to filter the artifacts, the freely moving rats were hosted in a Faraday cage. The rats were monitored by a motion sensitive video-monitoring system for 2-4 weeks after the appearance of spontaneous seizures to observe and evaluate behavioral seizure types. The obtained data was analyzed in offline mode, manually. Epileptic discharges were defined as spikes with a frequency of at least of 5 $\mathrm{Hz}$ and with amplitude at least twice as the baseline. If an electrographic seizure was recorded, we analyzed the video-monitoring of the respective period as well. Behavioral changes during kindling were scored according to the Racine scale [11].

Descriptive statistics and nonparametric tests (twotailed Mann-Whitney) were applied for data analysis using GraphPad Prism 5.00 for Windows (GraphPad Software, Inc., San Diego, California, USA). Statistical data, unless otherwise specified, is presented as Mean $\pm \mathrm{SD}$. We considered a change statistically significant at $\mathrm{p}<0.05$.

\section{Results}

We recorded a baseline trace in all rats from both groups. Recordings on freely moving animals were possible without electrical artifacts. Frequency analysis of the recorded traces showed marked theta $(4-7 \mathrm{~Hz})$ rhythm on both hippocampus electrodes (Figure 1).

The corticogram recorded from animals that were not stimulated (i.e. animals from the sham operated group) did not show electrical activity characteristic for epilepsy.
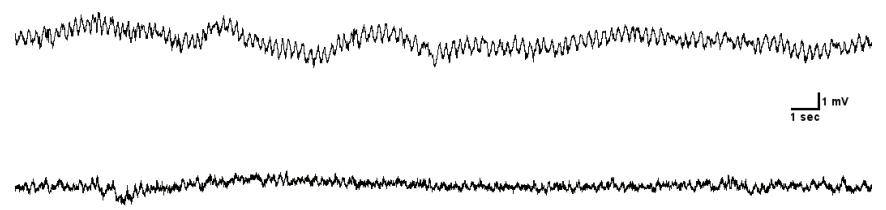

Fig. 1. Baseline electrical activity recorded in freely moving rat. Traces from top to bottom: left hippocampus, right hippocampus, left premotor area, right premotor cortex.

We repeatedly (daily) stimulated 4 rats (kindled group) eliciting seizures (Figure 2). The initial ADT was $350 \mu \mathrm{A} \pm 50 \mu \mathrm{A}$ but after 6 days of stimulation it was reduced to $200 \mu \mathrm{A} \pm 25 \mu \mathrm{A}$, which did not change further. The average seizure severity on the Racine scale was $2.6 \pm 0.4$ by day 6 , this gradually increased to $4.4 \pm 0.6$ by day 20 .

The first spontaneous seizures started to appear after 31 days of stimulation (Figure 3). All stimulated rats developed secondary seizures or spontaneous seizures. In $71 \%$ of the cases seizures started from the left hippocampus then propagated to the right hippocampus and then to the cortex. In case of spontaneous seizures the preictal 


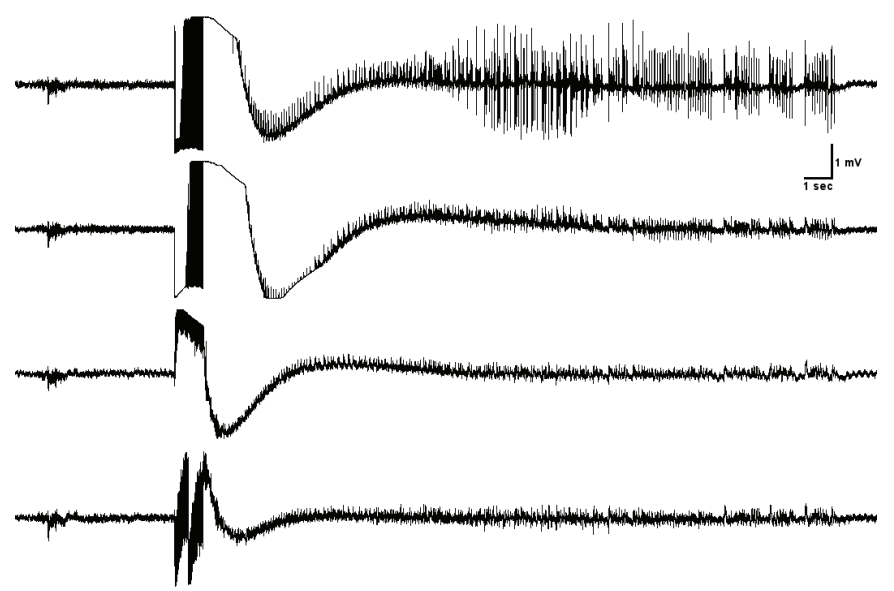

Fig. 2. Epileptic seizure initiated by a train of stimuli. The seizure was initiated in the left hippocampus and propagated bilaterally to the cortex. Note the spontaneous bursting activity before stimulation. The order of traces is similar to Figure 1.

spike amplitude decreased gradually but without statistical significance whereas the full width at half maximum increased gradually from $51 \pm 4$ msec to $110 \pm 5 \mathrm{msec}(\mathrm{p}<$ $0.05)$ during the same seizure. The amplitude of the negative field potential deflection (NFPD) that characterized the preictal spikes increased by $62 \%(\mathrm{p}<0.05)$ until the start of the epileptic discharges.

In all cases we confirmed by Nissl staining the correct position of the stimulation electrode.

\section{Discussion}

During epileptogenesis the central nervous system suffers major structural and functional changes that includes, but it is not limited to, cellular death, synaptic reorganization, axonal sprouting, gliosis, neurogenesis and pathological synchronization, which together lead to hyperexcitability [12]. In order to study epilepsy there are many experimental methods [13], that can be grouped in two major categories: in vitro and in vivo studies. In vitro studies have several major limitations, such as they do not characterize the progressive nature of the epileptogenesis and they do not offer reliable data about the interaction of neuronal networks. These limitations can be overcome by in vivo chronic studies. The most frequently used in vivo studies employ a chemically and electrically induced status epilepticus, but these studies have their limitations as well: high mortality of animals, controversies relating the human relevance of the epileptogenesis in rats with a severely damaged neuronal network, the high number of daily seizures that denotes a very severe epilepsy and creates a high degree of discomfort to the animals [14]. Kindling studies offer an alternative that may overcome the limitations of other studies. It creates a local (i.e. focal) electrical activation and a slower epileptogenesis that is more characteristic to human TLE [15] and allows to observe both the propagation of the epileptiform discharges and the interictal periods [16].

The kindling model that we used creates only a limited lesion that induces, at first, stimulated focal electrographic seizures, then stimulated secondarily generalized seizures, and in time may lead to spontaneous recurrent seizures that are progressive in nature (both regarding severity and frequency of seizures) [14].

The critical role of the amygdala in epilepsy is known for quite a while [7] and it is also known that it has the lowest kindling induction threshold in the limbic system $[17,18]$. The amygdala kindling model is considered an important tool in assessing TLE and can be used to assess the progression and generalization of limbic seizures [19]. The lateral nuclei of the left amygdala were chosen because of their reciprocal connections with the hippocampus [20] and because of its anxiolytic effect [21].

The current intensity for obtaining ADT in our animals is consistent with previous findings [7,22]. The gradual reduction of ADT was described earlier [23] and it may be related to histological [24], neurochemical [25] changes

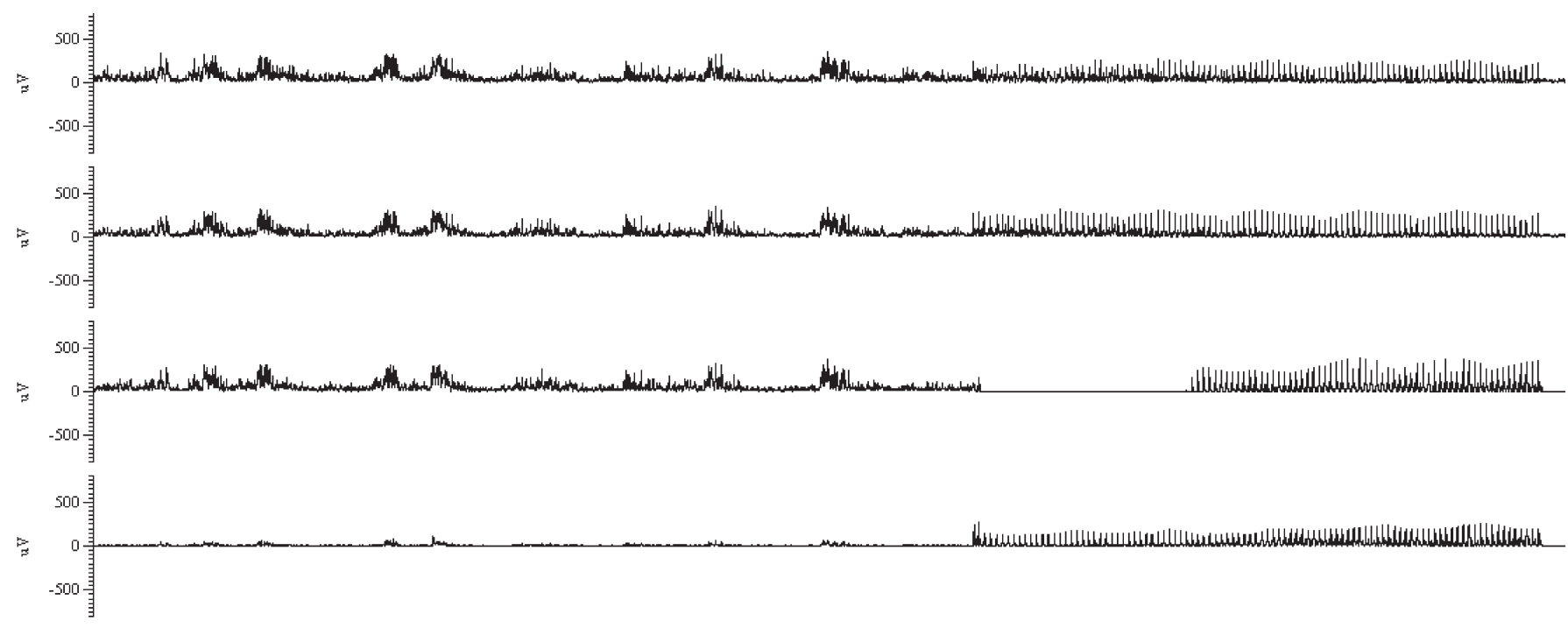

Fig. 3. Spontaneous epileptic seizure recorded in freely moving rat. Note the generalized bursting activity that precedes the seizure. The order of traces is similar to Figure 1. 
or to microhemorrhages and the deposition of iron [26]. The gradual reduction of the level of ADT can actually be observed as a consequence of the electrode implantation, but this was observed only after longer periods [23, 27]. As such in our case the reduction of ADT was a consequence of repetitive stimulation [22].

The electrographical progression of seizures was typical in our rats. The initial stimulation triggered a simple, short mono- or biphasic afterdischarge that did not propagate to the controlateral hemisphere. With repetitions, the electrical activity increases in complexity and projects invariably to distant neuroanatomic sites, including the controlateral hemisphere [28].

Kindling evokes a brief network synchronization that induces progressive functional alterations [29]. This feature indicates that kindling is a form of activity dependent, seizure-induced plasticity, which induces a permanently increased seizure susceptibility [30]. The repetitive nature of stimulation creates progressive functional and structural alterations that include altered synaptic transmission [31], gene expression induction [32], apoptotic neuronal loss [33], neurogenesis [34] and glial proliferation [35]. These changes eventually will lead to a significant reduction of GABAergic interneurons $[36,37]$ that can explain the increase of the severity of the seizures in our animals and the appearance of spontaneous seizures.

In our data from freely moving rats we found a significant gradual increase of preictal spike duration before the start of seizure like events. This change was already revealed in several, albeit in vitro, models of epilepsy that showed a marked inward current, with gradually increasing duration, preceding the start of the seizures [38, 39]. Therefore the appearance of seizures might be a result of a longer depolarization that can be associated with pathological oscillations. In our data the significant increase of the amplitude of NFPD, as we approach the start of the seizure, can actually reflect a better synchronization of synaptic inputs that allows for pathological high frequency oscillations and propagation of epileptic discharges [40].

\section{Conclusion}

Our study confirmed that the progression of temporal lobe epilepsy, as seen in humans, can be reproduced with high fidelity in the kindling model. This study confirms in vivo the increase in preictal spike duration as well as the increase of the amplitude of NFPD during the preictal period that reflects the oncoming pathological synchronization, which may allow the initiation of epileptic discharges and the propagation of the electrical activity from the limbic system to the cortex.

\section{Limitations of the study}

The results of the present study should be interpreted with care as the small number of animals hinders the possibility to have a strong statistical evidence.

\section{Acknowledgements}

This work was supported by the Internal Grant System of the University of Medicine and Pharmacy Tîrgu Mureş, contract number CIGCS 12/2013 as well as CNCSIS TD 114/2007. Károly Orbán-Kis was supported under the frame of European Social Found, Human Resources Development Operational Programme 2007-2013, project no. POSDRU/159/1.5/S/136893.

\section{References}

1. Smith PE. Introduction: The causes of epilepsy. Epilepsia. 2012 Sep;53 Suppl 4:1-2

2. Bertram E. The relevance of kindling for human epilepsy. Epilepsia. 2007;48 Suppl 2:65-74.

3. Mazarati A, Bragin A, Baldwin R et al. Epileptogenesis after self-sustaining status epilepticus. Epilepsia. 2002;43 Suppl 5:74-80.

4. Milner B, Squire LR, Kandel ER. Cognitive neuroscience and the study of memory. Neuron. 1998 Mar;20(3):445-468.

5. Montgomery SM, Buzsaki G. Gamma oscillations dynamically couple hippocampal CA3 and CA1 regions during memory task performance. Proc Natl Acad Sci U S A. 2007 Sep 4;104(36):14495-14500.

6. Tellez-Zenteno JF, Hernandez-Ronquillo L. A review of the epidemiology of temporal lobe epilepsy. Epilepsy Res Treat. 2012;2012:630853.

7. Goddard GV, Mclntyre DC, Leech CK. A permanent change in brain function resulting from daily electrical stimulation. Exp Neurol. 1969 Nov;25(3):295-330.

8. McNamara JO. Kindling: an animal model of complex partial epilepsy. Ann Neurol. 1984;16 Suppl:S72-76.

9. Sutula TP. Mechanisms of epilepsy progression: current theories and perspectives from neuroplasticity in adulthood and development. Epilepsy Res. 2004 Jul-Aug;60(2-3):161-171.

10. Paxinos G, Watson C. The Rat Brain in Steriotaxic Coordinates: Academic Press: San Diego; 1997.

11. Racine RJ. Modification of seizure activity by electrical stimulation. II. Motor seizure. Electroencephalogr Clin Neurophysiol. 1972 Mar;32(3):281-294.

12. Goldberg EM, Coulter DA. Mechanisms of epileptogenesis: a convergence on neural circuit dysfunction. Nat Rev Neurosci. 2013 May;14(5):337-349.

13. Orbán-Kis K, Metz J, Szilágy T. Állatmodellek jelentősége az epilepszia kutatásában. Orvostudományi Értesítő. 2008;81:88-91.

14. Dichter MA. Models of epileptogenesis in adult animals available for antiepileptogenesis drug screening. Epilepsy Res. 2006 Jan;68(1):31-35.

15. Curia G, Lucchi $\mathrm{C}$, Vinet $\mathrm{J}$ et al. Pathophysiogenesis of mesial temporal lobe epilepsy: is prevention of damage antiepileptogenic? Curr Med Chem. 2014;21(6):663-688.

16. Morimoto K, Fahnestock M, Racine RJ. Kindling and status epilepticus models of epilepsy: rewiring the brain. Prog Neurobiol. 2004 May;73(1):160.

17. Kandratavicius L, Balista PA, Lopes-Aguiar C et al. Animal models of epilepsy: use and limitations. Neuropsychiatr Dis Treat. 2014;10:16931705.

18. Loscher W. Animal models of intractable epilepsy. Prog Neurobiol. 1997 Oct;53(2):239-258.

19. McIntyre DC, Kelly ME, Dufresne C. FAST and SLOW amygdala kindling rat strains: comparison of amygdala, hippocampal, piriform and perirhinal cortex kindling. Epilepsy Res. 1999 Jul;35(3):197-209.

20. Pitkanen A, Savander V, LeDoux JE. Organization of intra-amygdaloid circuitries in the rat: an emerging framework for understanding functions of the amygdala. Trends Neurosci. 1997 Nov;20(11):517-523.

21. Adamec R, Blundell J, Burton P. Anxiolytic effects of kindling role of anatomical location of the kindling electrode in response to kindling of the right basolateral amygdala. Brain Res. 2004 Oct 22;1024(1-2):44-58.

22. Morales JC, Alvarez-Ferradas $\mathrm{C}$, Roncagliolo $\mathrm{M}$ et al. A new rapid kindling variant for induction of cortical epileptogenesis in freely moving rats. Front Cell Neurosci. 2014;8:200.

23. Ng MS, Hwang P, Burnham WM. Afterdischarge threshold reduction in the kindling model of epilepsy. Epilepsy Res. 2006 Dec;72(2-3):97-101.

24. Robinson N, Duncan P, Gehrt M et al. Histochemistry of trauma after electrode implantation and stimulation in the hippocampus. Arch Neurol. 1975 Feb;32(2):98-102

25. Ben Attia M, N'Gouemo P, Belaidi M et al. Kindling and electrode effects on the benzodiazepine receptors density of olfactory bulb and hippocampus after olfactory bulb kindling. Neurosci Lett. 1992 Aug 31;143(1-2):74-78. 
26. Loscher W, Wahnschaffe U, Honack D, Rundfeldt C. Does prolonged implantation of depth electrodes predispose the brain to kindling? Brain Res. 1995 Oct 30;697(1-2):197-204.

27. Loscher W, Horstermann D, Honack D et al. Transmitter amino acid levels in rat brain regions after amygdala-kindling or chronic electrode implantation without kindling: evidence for a pro-kindling effect of prolonged electrode implantation. Neurochem Res. 1993 Jul;18(7):775-781.

28. Mclntyre DC. The kindling phenomenon. In: Pitkanen A, Schwartzkroin PA, Moshe SL, editors. Models of seizure and epilepsy. Unites States of America: Elsevier Academic Press; 2005. p. 351-364.

29. Golarai G, Cavazos JE, Sutula TP. Activation of the dentate gyrus by pentylenetetrazol evoked seizures induces mossy fiber synaptic reorganization. Brain Res. 1992 Oct 16;593(2):257-264.

30. Sutula TP, Ockuly J. Kindling, Spontaneous Seizures, and the Consequences of Epilepsy: More Than a Model. In: Pitkanen A, Schwartzkroin PA, Moshe SL, editors. Models of seizure and epilepsy. Unites States of America: Elsevier Academic Press; 2005. p. 395-406.

31. Lynch M, Sayin U, Golarai G, Sutula T. NMDA receptor-dependent plasticity of granule cell spiking in the dentate gyrus of normal and epileptic rats. J Neurophysiol. 2000 Dec;84(6):2868-2879.

32. He XP, Kotloski R, Nef $S$ et al. Conditional deletion of TrkB but not BDNF prevents epileptogenesis in the kindling model. Neuron. $2004 \mathrm{Jul}$ 8;43(1):31-42.

33. Kotloski R, Lynch M, Lauersdorf S, Sutula T. Repeated brief seizures induce progressive hippocampal neuron loss and memory deficits. Prog Brain Res. 2002;135:95-110.

34. Scharfman HE, Sollas AL, Goodman JH. Spontaneous recurrent seizures after pilocarpine-induced status epilepticus activate calbindinimmunoreactive hilar cells of the rat dentate gyrus. Neuroscience. 2002;111(1):71-81.

35. Szyndler J, Wierzba-Bobrowicz T, Maciejak P et al. Pentylenetetrazolkindling of seizures selectively decreases $[3 \mathrm{H}]$-citalopram binding in the CA-3 area of rat hippocampus. Neurosci Lett. 2002 Dec 19;335(1):49-53.

36. Sayin U, Osting S, Hagen J et al. Spontaneous seizures and loss of axoaxonic and axo-somatic inhibition induced by repeated brief seizures in kindled rats. J Neurosci. 2003 Apr 1;23(7):2759-2768.

37. Szilagyi T, Szava I, Metz EJ et al. Untangling the pathomechanisms of temporal lobe epilepsy-The promise of epileptic biomarkers and novel therapeutic approaches. Brain Res Bull. 2014 Oct;109C:1-12.

38. Borck C, Jefferys JG. Seizure-like events in disinhibited ventral slices of adult rat hippocampus. J Neurophysiol. 1999 Nov;82(5):2130-2142.

39. Lee AC, Wong RK, Chuang SC et al. Role of synaptic metabotropic glutamate receptors in epileptiform discharges in hippocampal slices. J Neurophysiol. 2002 Oct;88(4):1625-1633.

40. Nyikos L, Lasztoczi B, Antal K et al. Desynchronisation of spontaneously recurrent experimental seizures proceeds with a single rhythm. Neuroscience. 2003;121(3):705-717. 